\title{
TPT1/ TCTP-regulated pathways in phenotypic reprogramming
}

\author{
Robert Amson ${ }^{1}$, Salvatore Pece ${ }^{2,3,4}$, Jean-Christophe Marine ${ }^{5,6}$, \\ Pier Paolo Di Fiore ${ }^{2,3,4}$, and Adam Telerman ${ }^{1}$
}

\author{
${ }^{1}$ CNRS-UMR 8113, LBPA, École Normale Supérieure, 61 avenue du Président Wilson, 94235 Cachan, France \\ ${ }^{2}$ Istituto FIRC di Oncologia Molecolare, Via Adamello 16, 20139, Milan, Italy \\ ${ }^{3}$ Istituto Europeo di Oncologia, Via Ripamonti 435, 20141, Milan, Italy \\ ${ }^{4}$ Dipartimento di Medicina, Chirurgia ed Odontoiatria, University of Milan, 20122, Milan, Italy \\ ${ }^{5}$ Laboratory for Molecular Cancer Biology, Center for the Biology of Disease, VIB, 3000 Leuven, Belgium \\ ${ }^{6}$ Laboratory for Molecular Cancer Biology, Center of Human Genetics, KULeuven, 3000 Leuven, Belgium
}

\begin{abstract}
Evolutionary conserved and pleiotropic, the TPT1/TCTP gene (translationally controlled tumor protein, also called HRF, fortilin), encodes a highly structured mRNA shielded by ribonucleoproteins and closely resembling viral particles. This mRNA activates, as do viruses, protein kinase $\mathbf{R}$ (PKR). The TPT1/TCTP protein is structurally similar to mRNA-helicases and MSS4. TPT1/TCTP has recently been identified as a prognostic factor in breast cancer and a critical regulator of the tumor suppressor p53 and of the cancer stem cell (SC) compartment. Emerging evidence indicates that TPT1/TCTP is key to phenotypic reprogramming, as shown in the process of tumor reversion and possibly in pluripotency. We provide here an overview of these diverse functions of TPT1/TCTP.
\end{abstract}

\section{Initial discoveries}

As many proteins of unknown function, TPT1/TCTP was initially named according to its molecular weight as Q23 [1], P21 [2], or P23 [3]. Subsequently, TPT1/TCTP was characterized as the histamine releasing factor (HRF) and studied for its extrinsic function in allergy and inflammation [4-7].

The relevance of TPT1/TCTP to tumorigenesis [8] was derived from two converging lines of investigation. The first concerned the function of TSAP6, a direct transcriptional target of the tumor suppressor p53 encoding a 6transmembrane domain protein that regulates the cell cycle, apoptosis, and iron metabolism [9-12]. TPT1/TCTP was found to interact with TSAP6, which - in turn regulated the secretion of TPT1/TCTP via the non-classical pathway, including exosomes $[13,14]$. The role of the p53TSAP6 axis in regulating protein secretion was further highlighted [15]. A second line of investigation approached the issue of tumor reversion by deriving revertant cells from human leukemia cell lines and solid tumors [16-19]. To trace the molecular events leading to reversion, differential gene expression between the parental tumor cells and the revertants was investigated and TPT1/TCTP was found to represent the most differentially expressed

Corresponding author: Telerman, A. (telerman@noos.fr,

adam.telerman@lbpa.ens-cachan.fr).

Keywords: p53; MDM2; tumor reversion; stem cells. (underexpressed) gene [18]. Subsequent work established that the reduction in TPT1/TCTP levels was indeed responsible for the reprogramming of cancer cells into revertants that lost most of their malignant phenotype [18,19].

The outlook on TPT1/TCTP acquired an even more complex dimension when it was found that, in Drosophila, $T P T 1 / T C T P$ is epistatic to TSC1 and dRheb in regulation of the mechanistic target of rapamycin (mTOR) pathway [20]. In addition, TPT1/TCTP was found to be an upstream activator of oct4 and nanog in somatic nuclei transplantation experiments [21]. There seems to be convergent evidence, therefore, for an important role of TPT1/TCTP in the reprogramming of various phenotypes, a concept underscored by the wide variety of protein-protein interactions engaged by TPT1/TCTP (Figure 1) that witness or predict an involvement in diverse processes such as cell survival, proliferation and growth, inflammation, DNA damage sensing/repair, and RNA/ribosome/protein biogenesis. Some of the characteristics of TPT1/TCTP are reminiscent of viral features, such as the highly structured mRNA that activates the interferon-inducible PKR [22], the ribonucleoprotein (RNP) particles surrounding this mRNA [2], the structural similarity between TPT1/TCTP protein and helicases, the way it antagonizes P53 [23], and its secretion by cells through exosomes [13,14], as is the case for HIV [24,25]. In this review, we will focus on numerous structural, evolutionary, and functional properties of TPT1/TCTP that highlight the concept that this protein might sit at the heart of important reprogramming networks that impact several cellular programs of physiological and pathological relevance.

\section{TPT1/TCTP mRNA activates PKR}

Initial attention to TPT1/TCTP, at the beginning of the 1980 s, was directed not so much at its protein function, but rather to the regulation, and to possible functions, of its mRNA. TPT1/TCTP was originally discovered as a protein inducible by serum activation of quiescent cells [1]. The fact that its induction was insensitive to actinomycin $\mathrm{D}$ argued for regulation at the post-transcriptional level [1]. A possible mechanism for such regulation emerged shortly thereafter, when TPT1/TCTP was identified as one of a small series of untranslated messengers in a 'masked state' 


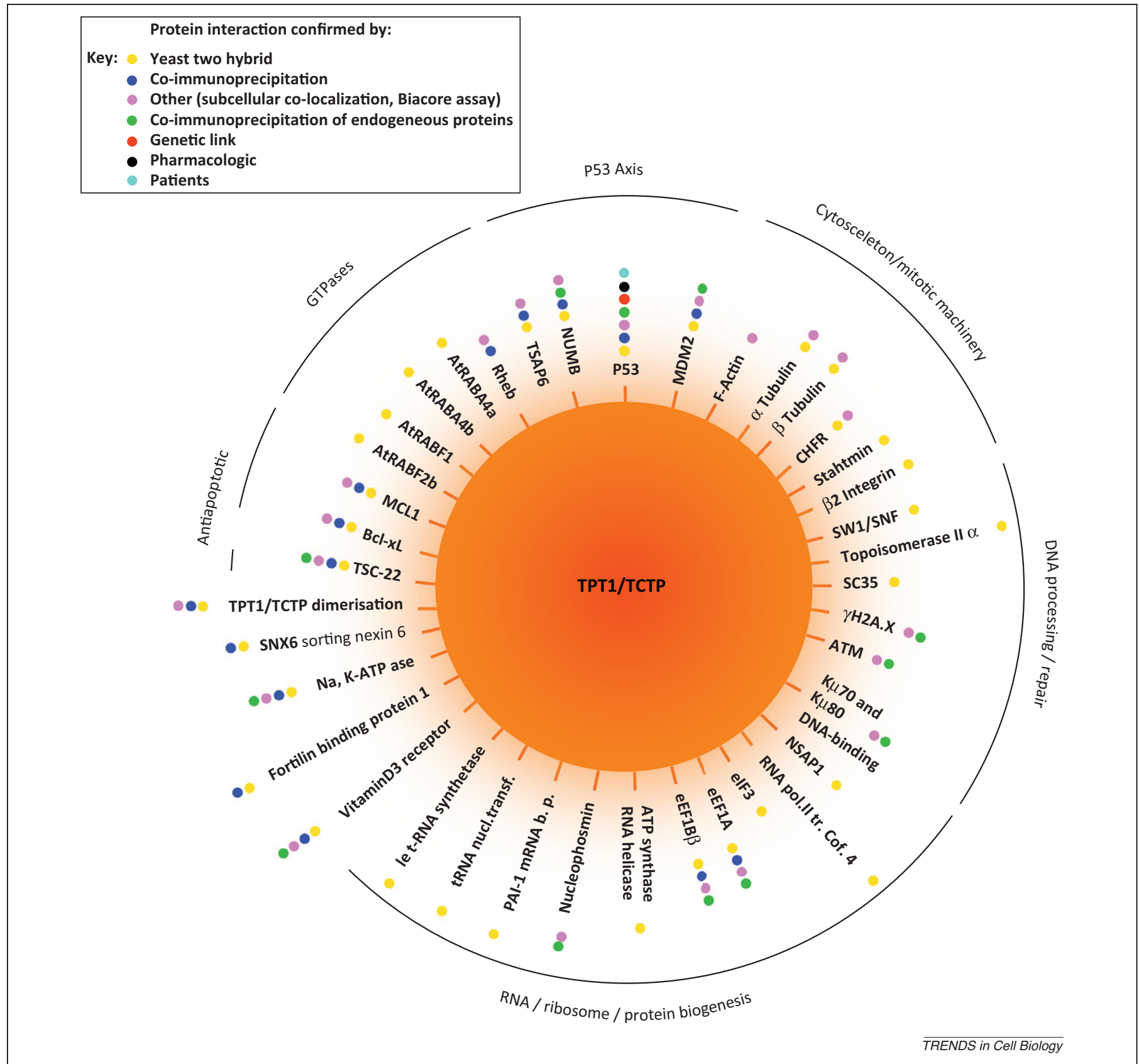

Figure 1. Schematic representation of the proteins interacting with TPT1/TCTP. Most of these proteins were found by yeast two-hybrid screening (yellow) and for some the interactions were confirmed by coimmunoprecipitation of overexpressed proteins (blue), subcellular colocalization, Biacore and other assays such as pull-down (pink), immunoprecipitation of endogenous cellular proteins (green), genetic interactions in murine models (red), use of pharmacological agents (black), or clinical relevance in a

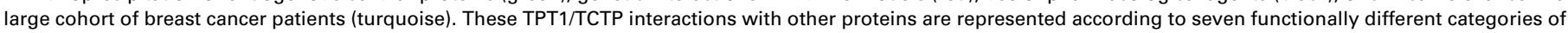
proteins; namely, the $\mathrm{P} 53$ axis, cytoskeleton and mitotic machinery, DNA processing and repair, RNA/ribosome and protein biogenesis, other proteins such as the Na, $\mathrm{K}$ ATPase, antiapoptotic proteins, and GTPase-related proteins. A series of these interactions have been reported (https://usgene.sequencebase.com/patents/US20060140970) [79] including: Stahtmin/OP18, $\beta 2$-integrin, DNA helicase SW1/SNF, topoisomerase II $\alpha$, splicing factor SC35, non structural protein 1 (NS1) of the minute virus of mice, RNA polymerase II transcription cofactor 4, translation initiation factor 3 (elF3), ATP synthase DEAE/H RNA helicase, PAI-1 mRNA-binding protein, tRNA nucleotidyl transferase, and isoleucine t-RNA synthetase.

associated with small RNP particles [2]. Typically, these masked messengers become active after deproteinization, a situation reminiscent of the life cycle of viruses.

Although it was understood at the end of the 1980s that the decay of mRNAs is partially determined by the structure of their $3^{\prime}$ untranslated region (UTR), which folds into hairpins and functions as a barrier against $3^{\prime}$ exonuclease [26], it was only in 2002 that it was discovered that the mRNA for TPT1/TCTP is potentially highly structured
[22]. Indeed, the predicted secondary structure of the TPT1/TCTP mRNA suggests that there are multiple double-strand (ds) RNA domains (Figure 2). This finding allows us to draw another interesting parallel with viruses, in that dsRNA structures are known to activate the interferon-dependent PKR $[27,28]$, a property shared by the mRNA of TPT1/TCTP [22].

PKR is one of four kinases known to phosphorylate and thus inhibit the eukaryotic initiation factor eIF2 $\alpha$, thereby 


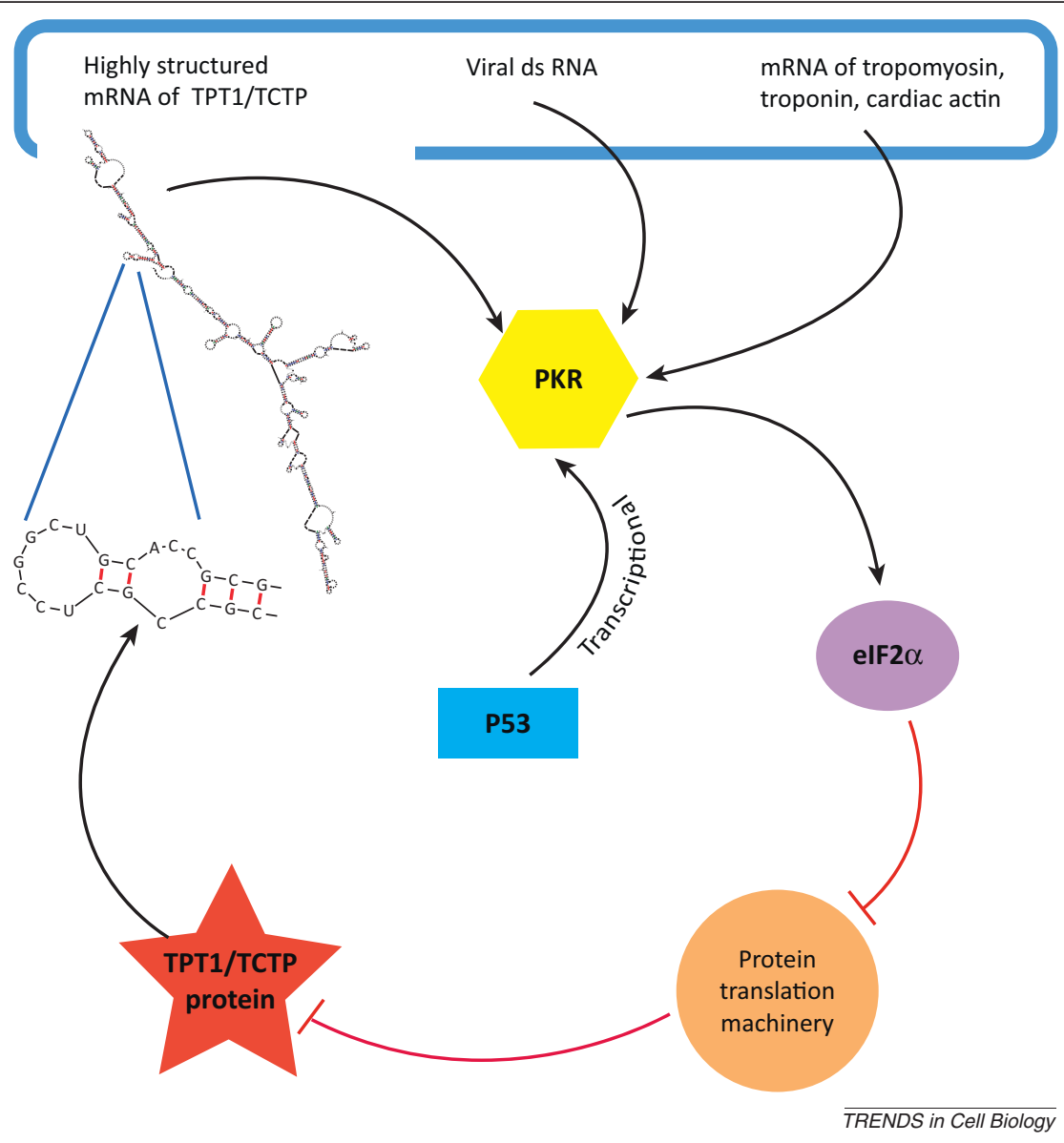

Figure 2. Activation of PKR by TPT1/TCTP mRNA. As described for viral double stranded (ds) RNAs [27,28], the mRNA for TCTP activates protein kinase R (PKR) [22], which is also the case for other mRNAs such as mRNA tropomyosin, mRNA troponin, and mRNA cardiac actin [31]. By adopting a highly structured conformation, TPT1/TCTP and other cellular mRNAs activate PKR [27]. PKR phosphorylates eukaryotic initiation factor 2 (elF2 $\alpha$ ), thereby stopping the protein translation machinery and inhibiting the translation of TPT1/TCTP. PKR is also directly activated at the transcriptional level by P53 [36].

halting the protein synthesis machinery. On viral infection, one of the major cellular responses comprises the recognition of viral dsRNA motifs by PKR. This, in turn, leads to PKR activation and ensuing phosphorylation of eIF2 $\alpha$. This virus-directed response constitutes only a facet of a much wider cellular program, because it is known that some cellular RNAs and endoplasmic reticulum stress can also activate PKR [29-35]. It was reported that the $3^{\prime}$ UTRs of the mRNAs of tropomyosin, troponin and, cardiac actin bind and activate PKR [29-31]. In the case of TPT1/TCTP, although the secondary structures involved have yet to be precisely mapped, both the $5^{\prime}$ and the $3^{\prime}$ UTR might assume ds configurations able to bind/activate PKR [22]. It must be pointed out, however, that according to the prediction of RNAfold (http://rna.tbi.univie.ac.at/cgi-bin/RNAfold.cgi) there are almost 1000 mRNAs that could potentially take a highly structured conformation. Together, these observations raise the possibility that TPT1/TCTP, like a number of other cellular proteins, may control protein synthesis by activating an interferon response. Although this is an exciting possibility, the physiological significance of these findings remains to be established. Notably, PKR was reported to be a direct transcriptional target of p53 [36], indicating that this tumor suppressor pathway may also be involved indirectly in the control of protein synthesis and of
TPT1/TCTP expression via this route (Figure 2). Thus, it seems reasonable to postulate that the presence of highly structured conformations might be necessary, but not sufficient, to bind/activate PKR. In turn, this calls for higher-resolution studies to clarify the mechanism and the physiological relevance of the control exerted by TPT1/TCTP mRNA over PKR.

\section{Structure-function analysis of TPT1/TCTP}

The structure of TPT1/TCTP was resolved by NMR spectroscopy (for the Schizosaccharomyces pombe protein), and by X-ray crystallography (for the human protein) [37,38]. This analysis revealed three domains that are highly conserved in TPT1/TCTP throughout phylogeny: (i) a core of nine $\beta$-strands including the short $\mathrm{H} 1$ helix; (ii) an $\alpha$ helical hairpin formed by two H2-H3 helices; and (iii) a mobile loop (Figure 3) [37,38]. There is no striking sequence homology with any other eukaryotic protein. However, the H2-H3 hairpin helices of TPT1/TCTP display both sequence homology and structural similarity with the H5-H6 helices of the bax-bcl2 family of proteins that are regulators of apoptosis (Figure 3) [38]. Importantly, TPT1/ TCTP acts as an antiapoptotic protein through a series of mechanisms $[18,38,39]$, including binding and potentiating Mcl1 [40,41] and Bclxl [42]. Of note, as in the case of the 


\section{TPT1/TCTP}
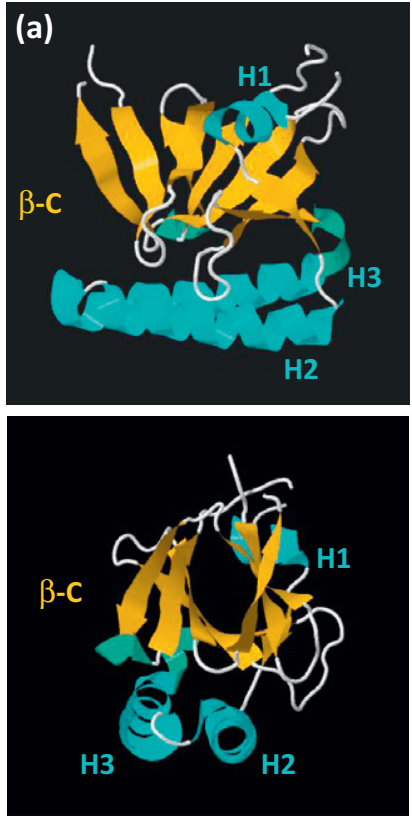

TPT1/TCTP - Bax
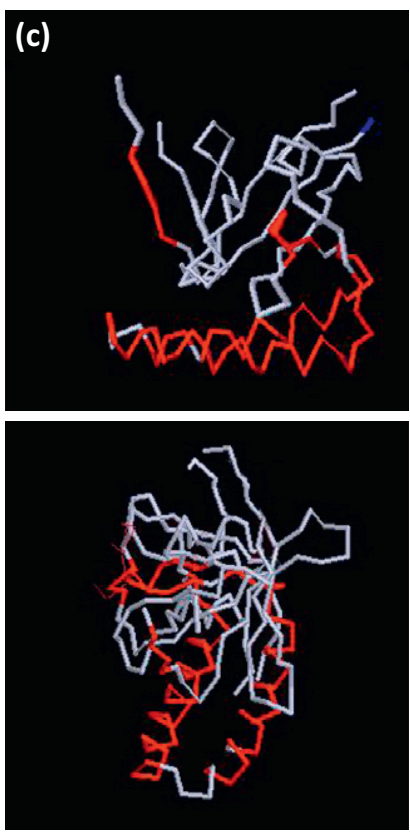
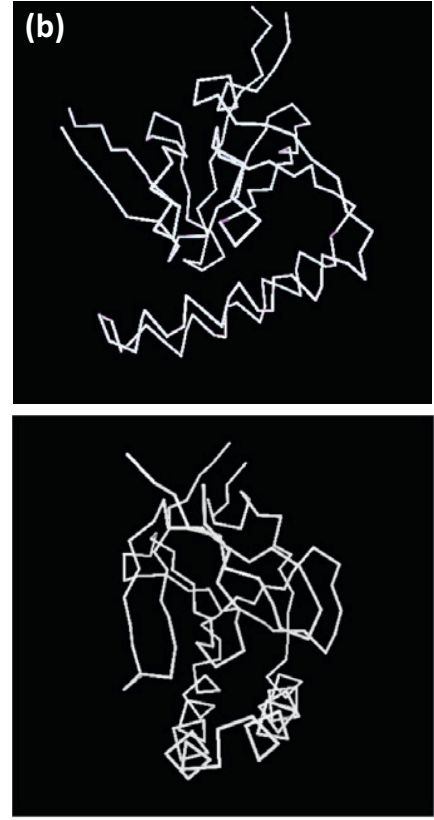

TPT1/TCTP - MSS4
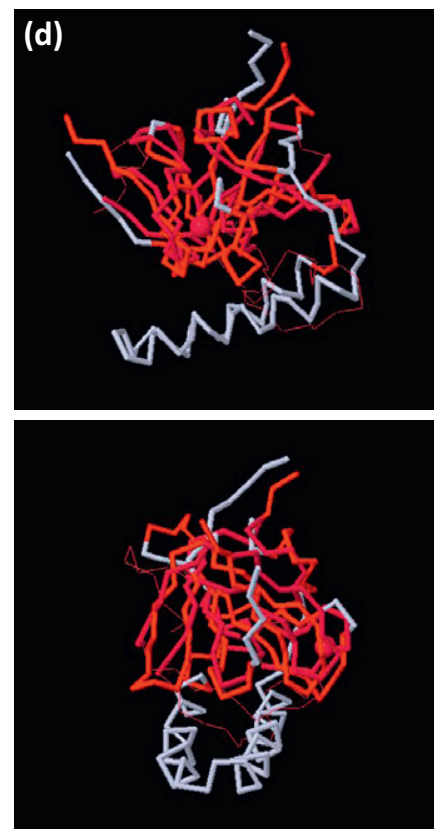

TPT1/TCTP -

RNA helicase (LGP2)
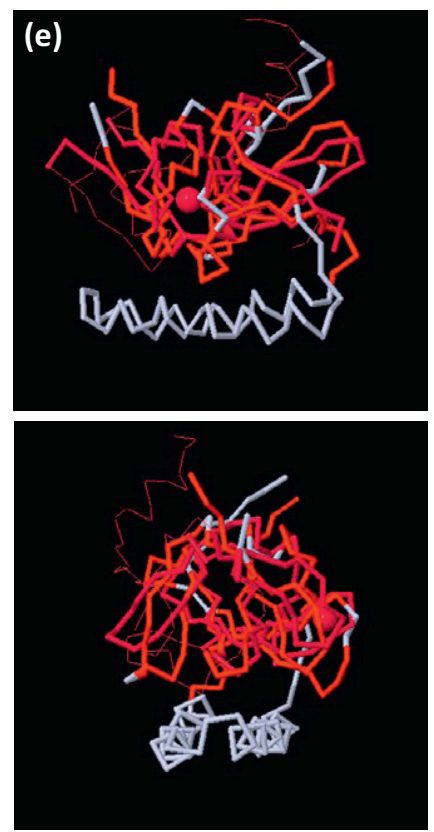

$\overline{T R E N D S ~ i n ~ C e l l ~ B i o l o g y ~}$

Figure 3. Structural homologies of TPT1/TCTP. Crystal structure of human TPT1/TCTP in two different orientations (upper and lower panels) [38]. (a) Ribbon representation; the helices $\mathrm{H} 1, \mathrm{H} 2$, and $\mathrm{H} 3$ are in turquoise and the core of nine $\beta$-strands $(\beta-\mathrm{C})$ in ochre. (b) Tube representation of the crystal structure of human TPT1/TCTP. (c) TPT1/TCTP and Bax. (d) TPT1/TCTP and MSS4. (e) TPT1/TCTP and the RNA helicase laboratory of genetics and physiology 2 (LGP2). For all representations, similar and superimposed structures are highlighted in red (http://ekhidna.biocenter.helsinki.fi/dali_server/).

H5-H6 hairpin of Bax, the H2-H3 hairpin helices of TPT1/ TCTP function as mitochondrial membrane-anchoring motifs to regulate membrane permeability and cytochrome $\mathrm{C}$ release during apoptosis. A Bax hybrid protein, in which the H5-H6 helices are replaced by the H2-H3 helices of TPT1/TCTP, retains its ability to induce apoptosis [38]. Mechanistically, transfection experiments support a model in which TPT1/TCTP antagonizes apoptosis by inserting into the mitochondrial membrane and inhibiting Bax homodimerization [38]. Moreover, this antiapoptotic role of TPT1/TCTP plays a crucial role in development, since TPT1/TCTP knockout in mice is embryonically lethal due to massive apoptosis $[38,43]$.

Despite the lack of major sequence homology to other proteins, the core domain of TPT1/TCTP displays remarkable structural similarity with two families of proteins: the 
methionine sulfoxide reductases and mammalian suppressor of yeast SEC4 (MSS4) [37]. We have recently searched for additional TPT1/TCTP protein structure similarities in other proteins using an in silico approach (http://ekhidna. biocenter.helsinki.fi/dali_server/) and uncovered a similarity with RNA helicases, such as the ATP-dependent RNA helicase laboratory of genetics and physiology 2 (LGP2) (Figure 3).

Methionine-R-sulfoxide reductase $\mathrm{B} 1$ protects against reactive oxygen species (ROS), which are generated during mitochondrial respiration and promote protein, lipid, and DNA damage. The high structural similarity between methionine-R-sulfoxide reductase B1 and TPT1/TCTP may indicate a common role in protecting cells against oxidative stress. Conversely, the homology with MSS4 has drawn much attention. MSS4 is a regulator of intracellular membrane trafficking that displays relatively low guanine nucleotide exchange factor (GEF) activity for Rabs. The similarity between MSS4 and TPT1/TCTP suggests that TPT1/TCTP could function as a GEF, a possibility that is further discussed below.

The structural similarity between the core domain of TPT1/TCTP and the mRNA helicases is striking (Figure 3). It has not been previously reported and deserves further comment. The RNA helicases are motor proteins involved not only in the unwinding of duplex nucleic acids, but also in protein-RNA interactions, initiation of translation, and ribosome biogenesis. The closest similarity is with the retinoic acid-inducible gene 1 (RIG1)-like receptors (RLRs). There are three RLR members: RIG-1, melanoma differentiation-associated factor 5 (MDA5), and LGP2 [44], a sensor of viral RNA, particularly ds viral RNAs $[45,46]$. RLR members activate signaling cascades that lead to the production of cytokines, such as type1 interferons [47]. This sensing of viral infection is the first barrier provided by the innate immune system [44]. Together with the previously described ability of TPT1/TCTP mRNA to activate the interferon-inducible PKR, these findings further raise the possibility that TPT1/ TCTP exerts its function, at least partly and through a different mechanism, by modulating interferon-regulated processes. This possibility warrants further experimental validation.

\section{TPT1/TCTP and mTOR signaling}

The first genetic link between TPT1/TCTP and components of the mTOR signaling pathway comes from experiments in flies [20]. The mTOR signaling pathway regulates diverse processes such as size and cell number by functioning as a sensor and integrator of environmental and intracellular signals [48]. Upstream of mTOR are nutrients, growth factors and their signaling pathways, energy, and stress. They ultimately regulate TSC1-TSC2, which acts as a GTPase-activating protein (GAP) for Rheb. When Rheb is in its GTP-bound form, it activates mTOR signaling. Whereas a null Drosophila dTCTP mutation is embryonically lethal at the first instar stage, knocking down dTCTP expression causes a reduction in overall eye and wing size [20] due to a decrease in both cell size and number. Importantly, $d T C T P$ was shown to be genetically epistatic to $d R h e b, T s c 1$, and $d \operatorname{Inr}[20]$.
In plants, Arabidopsis thaliana TPT1/TCTP (AtTCTP) is expressed widely, including during development [49,50]. Interestingly, AtTCTP regulates the duration of the cell cycle and thus mitotic but not postmitotic [50] growth. Although it was suggested that AtTCTP modulates the mTOR pathway, the mechanism remains to be identified.

The described studies argue that dTCTP is a positive regulator of the mTOR pathway, raising the question of the molecular mechanism. One possibility, based on the structural homology with MSS4, is that TPT1/TCTP might function as a GEF for Rheb. It was indeed shown that dTCTP has a weak GEF-like activity for dRheb [20]. These findings were, however, challenged shortly thereafter [5153]. This is perhaps unsurprising, because a similar debate has surrounded the putative GEF activity of MSS4 itself $[54,55]$. In this latter case, the issue was solved recently when resolution of the crystal structure of MSS4, in complex with Rab8, established the molecular basis of its weak and unconventional GEF activity for Rab GTPases [56]. Whether TPT1/TCTP is similarly endowed with weak GEF activity remains to be elucidated. If so, significant GEF activity in vivo might become apparent only under specific circumstances in which the protein is either expressed at high levels or specifically compartmentalized in the proximity of its putative targets, thus reaching a high local concentration. In this regard, we note that selective activation of the Rac1-GEF Tiam1 in a highly compartmentalized situation (endosomes) was recently demonstrated [57]. Regardless, the issue of how TPT1/TCTP might exert control over the mTOR pathway remains open.

\section{TPT1/TCTP in reprogramming: from SCs to tumor reversion}

The nuclei from differentiated cells can be reprogrammed when transferred into an egg or oocyte [58]. During this process, oct4 expression increases. Oct4 is a transcription factor that, when coexpressed with Sox2, c-MYC, and Klf4, can reprogram differentiated somatic cells into pluripotent embryo-like SCs. Strikingly, TPT1/TCTP was shown to regulate oct4 expression during nuclear transfer experiments [21]. Moreover, depletion of TPT1/TCTP reduces the transcription of both oct4 and nanog in undifferentiated cells. Of note, TPT1/TCTP interacts with nucleophosmin/ nucleoplasmin family member $1(\mathrm{Npm} 1)$ and decreases during differentiation of embryonic stem (ES) cells [5961]. Npm1 also binds oct4 during interphase in ES cells and may be involved in the differentiation process.

Tumor cells can undergo reprogramming and lose their malignant phenotype in a process referred to as tumor reversion [8]. To understand the molecular basis, revertants were derived from human tumor cells, ranging from leukemia cell lines to solid tumors [9,16-19,62-64]. These revertant cells barely formed tumors when injected into scid/scid mice, whereas the parental malignant cells form large tumors that rapidly killed the animals. By analyzing the gene expression profiles, over 300 genes were found to be differentially expressed between the tumor cells and revertants, including TPT1/TCTP [18], displaying 248 signals (equivalent to the amount of mRNA for TPT1/TCTP) in the tumor cells and only two in the revertant cells. It was 
further shown that silencing TPT1/TCTP in breast cancer cells allows reorganization into duct-like structures reminiscent of normal growth [18]. In line with this observation, inhibition of TPT1/TCTP expression in $v$-src-transformed NIH-3T3 cells results in a massive reversion of the malignant phenotype. To explore the function of TPT1/TCTP, a search for interacting proteins was performed and it was observed that TPT1/TCTP binds directly to the translation elongation factor eEF1A (which is a GTPase) and its guanine nucleotide exchange factor eEF1Bbeta [65]. This interaction between TPT1/TCTP and eEF1Bbeta has also been described by others [66]. TPT1/TCTP preferentially stabilized the GDP form of eEF1A and impaired the GDP exchange reaction promoted by eEF1Bbeta, indicating that TPT1/TCTP has guanine nucleotide dissociation inhibitor (GDI) activity [65]. This function of TPT1/TCTP as a GDI has been put forward as an argument against its GEF activity, but this might be an overinterpretation because eEF1A is a large G-protein. As mentioned above (Figure 1), TPT1/TCTP potentially interacts with a large series of proteins and it is striking to note that half of them are implicated in DNA damage sensing/repair and RNA/ribosome/protein biogenesis, which could be part of the program that switches off the malignant phenotype, an issue that warrants further investigation. This silencing of TPT1/TCTP, which is required for reprogramming tumor cells into revertants, raises the possibility that similar pathways that are at least partly controlled by TPT1/TCTP are implicated in the reprogramming of ES cells and cancer cells.

\section{The p53-TPT1/TCTP repressive feedback loop and 'stemness'}

The pleiotropic nature of the biological activities of TPT1/ TCTP is underscored by its ability to interact physically with an impressive series of protein partners participating in many different cellular functions [39-42,65,67-69] (Figure 1). One particularly relevant TPT1/TCTP interactor is the P53 tumor-suppressor protein. Although it was previously reported [18] that overexpression of P53 resulted in decreased levels of TPT1/TCTP, it was only recently demonstrated that TPT1/TCTP is a key regulator of P53 stability and activity [23] with possible consequences on the homeostasis of the $\mathrm{SC}$ compartment [23,70-75] (Box 1). Mechanistically, TPT1/TCTP downregulates P53 by promoting its ubiquitination by the E3ligase $\mathrm{Mdm} 2$ and subsequent proteasomal degradation [23] (Figure 4). This function of TPT1/TCTP is dependent on its ability to antagonize Numb [23], a major cell fate determinant involved in various developmental programs [76]. Numb has been shown to form a trimeric complex with P53 and Mdm2, and to inhibit Mdm2-mediated ubiquitination of P53, thereby stabilizing P53 levels and activity [77]. We demonstrated that TPT1/TCTP competes with Numb for binding to $\mathrm{Mdm} 2$, thereby overriding the inhibitory effect of Numb on Mdm2 and promoting P53 ubiquitination [23]. A second major mechanism by which TPT1/ TCTP promotes the proteasomal degradation of P53 is by directly stabilizing MDM2 and inhibiting its self-ubiquitination [23]. Interestingly, transcription of the TPT1/ TCTP gene is directly repressed by P53 (Figure 4). These

\section{Box 1. Breast cancer SCs}

Increasing evidence suggests that breast tumors, like the normal mammary gland, are hierarchically organized, containing a minority of cells (operationally definable as cancer SCs [CSCs]) endowed with 'stemness' properties, such as self-renewal, quiescence, extended replicative potential, and the ability to generate the heterogeneous, non-tumorigenic cell populations that constitute the tumor bulk [70]. Breast CSCs are thought to drive the initiation and maintenance of tumors, metastasis, and relapse. Selective elimination of these cells has therefore been proposed as an effective strategy for eradicating cancer. A better understanding of the molecular mechanisms regulating normal SC functions, and how these mechanisms are perturbed in cancer, is therefore vital to our understanding of breast tumorigenesis and to the development of more effective breast cancer therapies.

In this context, the tumor suppressor P53 is emerging as a critical regulator of $\mathrm{SC}$ homeostasis in different types of tissues, including the mammary gland [71]. Increased wild type (WT) p53 activity in the murine mammary gland leads to aberrant morphogenesis and reduction of the self-renewal potential of mammary SCs, demonstrated by serial transplantation experiments [72]. Conversely, the mammary epithelium of p53-deficient mice contains an increased number of SCs and displays increased susceptibility to tumorigenesis $[73,74]$. Consistently, it was recently demonstrated that downregulation of P53 following ErbB2 oncogene activation in the mammary epithelium is responsible for the aberrant self-renewal kinetics displayed by CSCs derived from ErbB2 mammary tumors [75]. Loss of p53 resulted in increased proliferation, unlimited selfrenewal potential, and a higher frequency of symmetric versus asymmetric cell divisions in CSCs, compared with their normal counterparts. Pharmacological restoration of P53 in ErbB2-CSCs using Nutlin-3, an inhibitor that prevents Mdm2-mediated ubiquitination and degradation of $\mathrm{P} 53$, correlated with restoration of asymmetric cell division, reduction in the number of CSCs in ErbB2 tumors, and delayed tumor growth. Notably, Nutlin-3 appeared to selectively target the CSC compartment, because negligible effects were observed in the bulk tumor cells and the normal mammary gland. Thus, P53 regulates the polarity of self-renewing divisions of mouse mammary SCs and loss of p53 promotes symmetric cell division, leading to expansion of the CSC compartment and tumor growth. Mechanisms impacting on p53 function are therefore likely to be involved in the subversion of SC homeostasis in breast carcinogenesis, as in the reciprocal repressive feedback loop between p53 and TPT1/TCTP [23]. The use of sertraline and thioridazine which target TPT1/TCTP and inhibit its function could lead to a new therapeutic route in cancer treatment $[19,23]$.

data indicate that P53 and TPT1/TCTP are engaged in reciprocal repression.

In vivo, this circuitry was explored by a mouse genetics approach [23] and received further support from the analysis of TPT1/TCTP expression in breast cancer patients (Box 2). In Tpt1/Tctp heterozygous mice, it was observed that P53 protein levels were readily detectable in virtually all tissues, in contrast with wt mice in which endogenous P53 levels are notoriously difficult to detect [23]. This observation supports a role for Tpt1/Tctp in the control of P53 stability under physiological conditions in vivo. Note that this increase in P53 steady-state levels is partly surprising, because a similar increase was not observed in Mdm2 heterozygous or even in Mdm2 hypomorphic mice, which express $30 \%$ of endogenous $\mathrm{Mdm} 2$ levels. This may be explained by the fact that P53 and Mdm2 are engaged in a classical negative feedback loop, whereas P53 and Tpt1/Tctp are in a reciprocal repression loop. As a consequence, although even slight differences in Tpt1/Tctp expression levels are expected to be amplified 


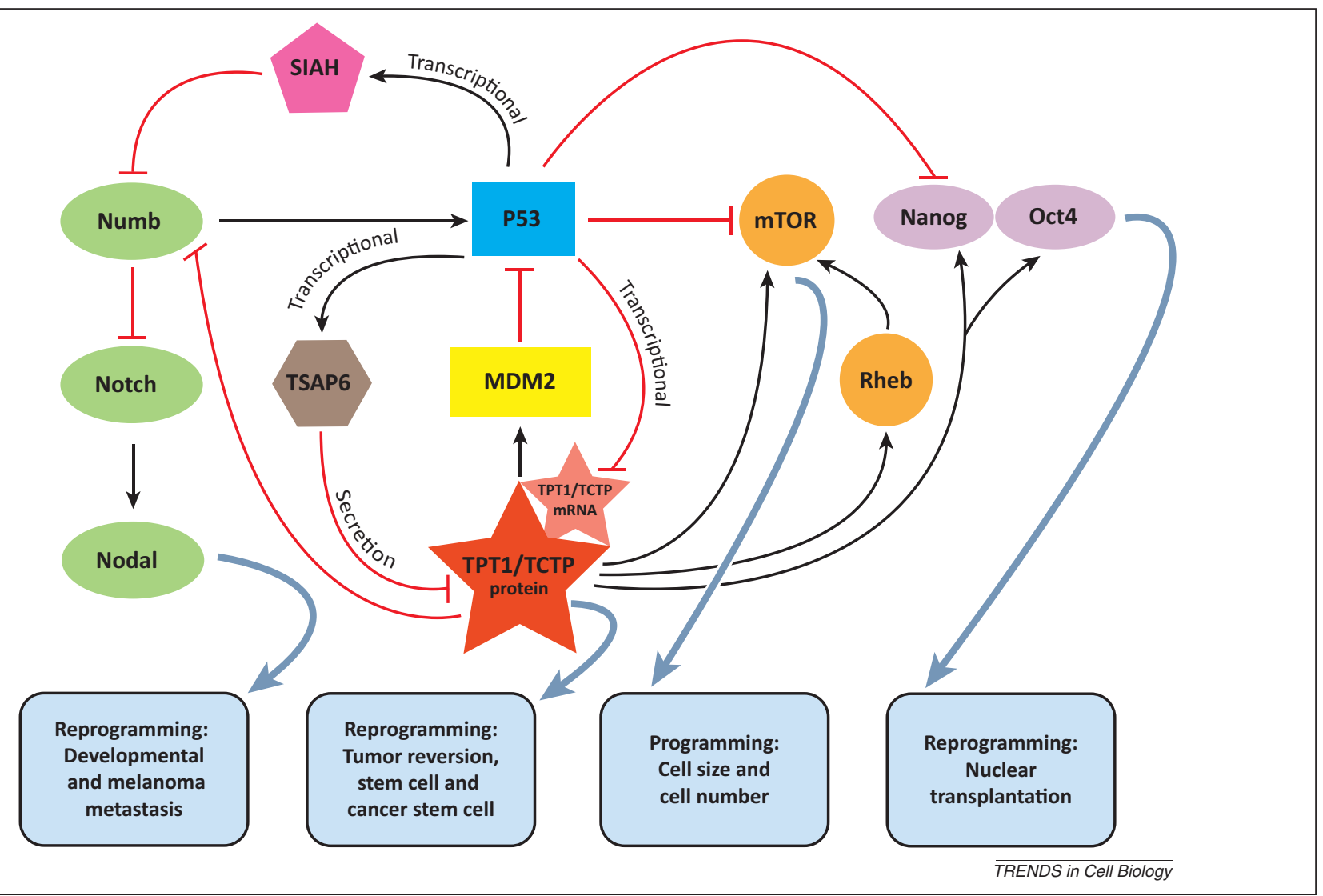

Figure 4. Converging reprogramming networks. TPT1/TCTP-NUMB-MDM2-P53 axis: TPT1/TCTP and P53 are engaged in a reciprocal repressive feedback loop [23]. TPT1/ TCTP promotes MDM2-dependant ubiquitination and degradation of P53. TPT1/TCTP binds and competes with NUMB [23], the latter stabilizing P53 [77]. P53 transcriptionally represses TPT1/TCTP and also activates TSAP6 [10], which regulates the non-classical secretion of proteins and promotes the secretion of TCTP via exosomes [13]. Furthermore, P53 activates the E3 ligase SIAH [80], which binds to NUMB and promotes its degradation. TPT1/TCTP-mTOR: Drosophila TPT1/TCTP regulates the mTOR pathway by acting as a guanine nucleotide exchange factor (GEF)-like molecule for Rheb [20]. Alternatively, TPT1/TCTP could activate mTOR by a different mechanism. P53 is a negative regulator of the mTOR pathway [81]. TPT1/TCTP-oct4-nanog: TPT1/TCTP is an upstream activator of oct4 and nanog in nuclear reprogramming [21]. Importantly oct4, when coexpressed with Sox2, c-MYC, and Klf4 in mouse embryonic and adult fibroblasts, reprograms them into induced pluripotent stem cells [82,83]. The transcription of nanog is repressed by P53 [84]. Numb-Notch-Nodal: the cell fate determinant Numb negatively regulates Notch [76]. Previous experiments showed that repression of Nodal expression is instrumental in reverting the malignant phenotype of melanoma cells [85] and recent evidence indicates that nodal is regulated by Notch in these processes [86]. Altogether, converging evidence points toward a central role for TPT1/TCTP in different reprogramming networks.

by the reciprocal repression loop, comparable variations in Mdm2 levels will be compensated instead by the negative feedback loop (Figure 4). Importantly, not only P53 basal levels are elevated in Tpt1/Tctp heterozygous thymocytes,

\section{Box 2. The TPT1/TCTP-P53 axis in breast cancer patients}

The TPT1/TCTP-P53 axis is also relevant to human breast cancer. In a cohort of 508 breast cancer patients, high TPT1/TCTP status correlated with clinical and pathological parameters of aggressive disease, such as a poor degree of differentiation, high tumor grade (G3), high proliferative activity, and negative estrogen receptor expression [23]. High TPT1/TCTP status was also an independent predictor of poor prognosis in these patients. TPT1/TCTP overexpression was frequently associated with a mutated p53 status, as expected based on the evidence that TPT1/TCTP is a negative transcriptional target of P53 [23]. This argues that, when p53 is dysfunctional due to primary inactivating mutations, the ensuing overexpression of TPT1/TCTP most likely represents a major event contributing to carcinogenesis. It is therefore not surprising that the negative regulation of P53 by TPT1/TCTP is counteracted by another cancer-relevant mechanism, namely the Numb/p53 circuitry, which is crucial sustaining p53 tumor-suppressor activity $[77,87]$. Interestingly, the biologically aggressive phenotype of TPT1/TCTP-overexpressing tumors is analogous to the clinically aggressive behavior of Numb-deficient breast tumors, in which deregulated Mdm2dependent degradation of P53 is also observed $[77,87]$. but irradiation (IR)-induced P53 stabilization is more pronounced in these cells, leading to increased P53 transcriptional activity and P53-dependent apoptosis [23]. These data demonstrate that Tpt1/Tctp haploinsufficiency sensitizes to P53-dependent apoptosis in vivo and therefore identify Tpt1/Tctp as a critical rate-limiting factor in the control of P53 stability and activity in response to genotoxic stress [23].

The consequences of the subversion of TPT1/TCTP-centered regulations were analyzed in breast cancer. TPT1/ TCTP was identified, via a comparative global transcriptome analysis of purified normal human mammary SCs, as one of the molecular traits characterizing the identity of normal human mammary SCs [78]. Direct immunofluorescence analysis of TPT1/TCTP expression confirmed the association of TPT1/TCTP with the normal human mammary SC compartment and its downregulation in differentiating progenitors [23]. These findings suggest that TPT1/ TCTP might be involved in the homeostasis of the mammary SC compartment, and - more specifically - that its downregulation is a prerequisite for the correct progression of precursors through the morphogenetic program. A corollary of this idea is that TPT1/TCTP expression must be tightly temporally and spatially regulated throughout 
morphogenesis, with aberrant TPT1/TCTP function possibly affecting the normal dynamics of the SC compartment. The first evidence in this direction came from the demonstration that silencing TPT1/TCTP expression in breast cancer cell lines (MCF7 and T47D) reverted the morphogenetically aberrant structures formed by these cells in 3D Matrigel cultures, restoring a growth pattern reminiscent of the outgrowths generated by normal mammary epithelial cells [18]. In addition, silencing of TPT1/TCTP in mouse mammary epithelial tumor cells derived from ErbB2 transgenic mice decreased the ability of these cells to generate mammospheres, an assay that measures the intrinsic SC content of a given epithelial cell population [23]. This effect of TPT1/TCTP on cancer SC self-renewal was accompanied by an increase in P53 levels, arguing that TPT1/TCTP influences SC function via the liaison with P53. Corroborating this notion, pharmacological inhibition of TPT1/TCTP using sertraline and thioridazine also resulted in increased p53 levels by counteracting Mdm2mediated ubiquitination of p53 and reduced the mammosphere-forming efficiency, thus providing stringent evidence for the existence of a TPT1/TCTP-p53 axis in the regulation of cancer SC self-renewal [23].

\section{Concluding remarks}

The sum of the reviewed evidence, which does not exhaust the multiplicity of biological processes in which TPT1/ TCTP is involved, argues that it sits at the heart of a number of programs of physiological relevance that control the modalities of cell growth in various contexts: from development to nutrient homeostasis, to stress responses and apoptosis, to self-renewal in SCs. Alterations of TPT1/ TCTP might therefore play a central role in cancer, as supported by increasing biological and molecular evidence. In this regard, we find particularly interesting observations that TPT1/TCTP-centered programs, involving regulation of the tumor suppressor p53, are involved in both subversion of the cancer SC compartment and the program of tumor reversion, arguing that the biological foundations of the latter might be represented by some form of molecular 'cure' of aberrant modalities of self-renewal of cancer SCs.

TPT1/TCTP has emerged as a critical survival factor and a regulator of cell fate determination. TPT1/TCTP regulates many different biological processes, all of which may converge to a limited set of key events that control stemness, pluripotency, and tumor reversion (Figure 4). In cancer, TPT1/TCTP could have an oncogenic function. Numerous features argue in favor of this possibility, including promoting cell growth, activating components of the mTOR pathway, inhibiting Bax homodimerization and apoptosis, antagonizing P53, and being expressed in aggressive breast cancers $[20,23,38]$. However, its overexpression in transgenic mice does not induce or favor tumor formation [6,69]. In addition, TPT1/TCTP mRNA is likely to activate PKR, which is in turn critical for the tumor suppressor function of P53 [36]. Although this function is not ascribable directly to TPT1/TCTP as a protein, it supports the notion that its increased transcription/expression might be part of tumor-suppressor circuitries. Maybe, like one of its protein partners, NPM1
[59-61], TPT1/TCTP combines both a tumor suppressive and an oncogenic activity that results in context-dependent cancer phenotypes. A recurrent theme is that only when TPT1/TCTP levels are decreased, notably in cancer, can cells be reprogrammed. Thus, TPT1/TCTP has the characteristics of a checkpoint - a switch necessary for reprogramming. The conservation of the TPT1/TCTP-converging networks (Figure 4) through phylogeny further underscores their relevance. Deciphering how all these functions are integrated and which biochemical and molecular mechanisms underscore their execution is the challenge lying ahead of us.

\section{Acknowledgments}

A.T. and R.A. dedicate this work to Hélène and Philippe Leloup and Béatrice Rosenberg, for their constant encouragement. We thank all our students, postdocs, and collaborators, for their involvement and contribution, and Michael Mathews for stimulating suggestions. Work in the authors' laboratories is supported by grants from the Agence Nationale de la Recherche Programme Blanc (ANR- 09-BLAN-0292-01), the European Union Network of Excellence CONTICANET, and the Association Sclérose Tubéreuse de Bourneville to A.T. and R.A.; the Associazione Italiana per la Ricerca sul Cancro (AIRC), MIUR (the Italian Ministry of University and Scientific Research), and the Italian Ministry of Health to S.P. and P.P.D.F.; the European Community (FP7, Mammastem Project), The Ferrari Foundation, the Monzino Foundation, and the CARIPLO Foundation to P.P.D.F.; the G. Vollaro Foundation to S.P.; and the Belgian Foundation against Cancer and FWO to J.C.M.

\section{References}

1 Thomas, G. et al. (1981) Transcriptional and translational control of cytoplasmic proteins after serum stimulation of quiescent Swiss 3T3 cells. Proc. Natl. Acad. Sci. U.S.A. 78, 5712-5716

2 Yenofsky, R. et al. (1982) Messenger RNA species partially in a repressed state in mouse sarcoma ascites cells. Proc. Natl. Acad. Sci. U.S.A. $79,5876-5880$

3 Bohm, H. et al. (1989) The growth-related protein P23 of the Ehrlich ascites tumor: translational control, cloning and primary structure. Biochem. Int. 19, 277-286

4 MacDonald, S.M. et al. (1995) Molecular identification of an IgEdependent histamine-releasing factor. Science 269, 688-690

5 Schroeder, J.T. et al. (1997) Recombinant histamine-releasing factor enhances IgE-dependent IL-4 and IL-13 secretion by human basophils. J. Immunol. 159, 447-452

6 Yeh, Y.C. et al. (2010) The effects of overexpression of histamine releasing factor (HRF) in a transgenic mouse model. PLoS ONE 5, e11077

7 Kashiwakura, J.C. et al. (2012) Histamine-releasing factor has a proinflammatory role in mouse models of asthma and allergy. $J$. Clin. Invest. $122,218-228$

8 Telerman, A. and Amson, R. (2009) The molecular programme of tumour reversion: the steps beyond malignant transformation. Nat. Rev. Cancer 9, 206-216

9 Amson, R.B. et al. (1996) Isolation of 10 differentially expressed cDNAs in p53-induced apoptosis: activation of the vertebrate homologue of the Drosophila seven in absentia gene. Proc. Natl. Acad. Sci. U.S.A. 93, 3953-3957

10 Passer, B.J. et al. (2003) The p53-inducible TSAP6 gene product regulates apoptosis and the cell cycle and interacts with Nix and the Myt1 kinase. Proc. Natl. Acad. Sci. U.S.A. 100, 2284-2289

11 Ohgami, R.S. et al. (2005) Identification of a ferrireductase required for efficient transferrin-dependent iron uptake in erythroid cells. Nat. Genet. 37, 1264-1269

12 Grandchamp, B. et al. (2011) A novel type of congenital hypochromic anemia associated with a nonsense mutation in the STEAP3/TSAP6 gene. Blood 118, 6660-6666

13 Amzallag, N. et al. (2004) TSAP6 facilitates the secretion of translationally controlled tumor protein/histamine-releasing factor via a nonclassical pathway. J. Biol. Chem. 279, 46104-46112 
14 Lespagnol, A. et al. (2008) Exosome secretion, including the DNA damage-induced p53-dependent secretory pathway, is severely compromised in TSAP6/Steap3-null mice. Cell Death Differ. 15, 1723-1733

$15 \mathrm{Yu}$, X. et al. (2006) The regulation of exosome secretion: a novel function of the p53 protein. Cancer Res. 66, 4795-4801

16 Telerman, A. et al. (1993) A model for tumor suppression using H-1 parvovirus. Proc. Natl. Acad. Sci. U.S.A. 90, 8702-8706

17 Nemani, M. et al. (1996) Activation of the human homologue of the Drosophila sina gene in apoptosis and tumor suppression. Proc. Natl. Acad. Sci. U.S.A. 93, 9039-9042

18 Tuynder, M. et al. (2002) Biological models and genes of tumor reversion: cellular reprogramming through tpt1/TCTP and SIAH-1. Proc. Natl. Acad. Sci. U.S.A. 99, 14976-14981

19 Tuynder, M. et al. (2004) Translationally controlled tumor protein is a target of tumor reversion. Proc. Natl. Acad. Sci. U.S.A. 101, 1536415369

20 Hsu, Y.C. et al. (2007) Drosophila TCTP is essential for growth and proliferation through regulation of dRheb GTPase. Nature 445, 785788

21 Koziol, M.J. et al. (2007) Tpt1 activates transcription of oct4 and nanog in transplanted somatic nuclei. Curr. Biol. 17, 801-807

22 Bommer, U.A. et al. (2002) The mRNA of the translationally controlled tumor protein P23/TCTP is a highly structured RNA, which activates the dsRNA-dependent protein kinase PKR. RNA 8, 478-496

23 Amson, R. et al. (2012) Reciprocal repression between P53 and TCTP. Nat. Med. 18, 91-99

24 Gould, S.J. et al. (2003) The Trojan exosome hypothesis. Proc. Natl. Acad. Sci. U.S.A. 100, 10592-10597

25 Nguyen, D.G. et al. (2003) Evidence that HIV budding in primary macrophages occurs through the exosome release pathway. J. Biol. Chem. 278, 52347-52354

26 Brawerman, G. (1987) Determinants of messenger RNA stability. Cell $48,5-6$

27 Tian, B. et al. (2004) The double-stranded-RNA-binding motif: interference and much more. Nat. Rev. Mol. Cell Biol. 5, 1013-1023

28 Garcia, M.A. et al. (2007) The dsRNA protein kinase PKR: virus and cell control. Biochimie 89, 799-811

29 Rastinejad, F. and Blau, H.M. (1993) Genetic complementation reveals a novel regulatory role for $3^{\prime}$ untranslated regions in growth and differentiation. Cell 72, 903-917

30 Davis, S. and Watson, J.C. (1996) In vitro activation of the interferoninduced, double-stranded RNA-dependent protein kinase PKR by RNA from the $3^{\prime}$ untranslated regions of human alpha-tropomyosin. Proc. Natl. Acad. Sci. U.S.A. 93, 508-513

31 Nussbaum, J.M. et al. (2002) The 3 '-untranslated regions of cytoskeletal muscle mRNAs inhibit translation by activating the double-stranded RNA-dependent protein kinase PKR. Nucleic Acids Res. 30, 1205-1212

32 Cuddihy, A.R. et al. (1999) Double-stranded-RNA-activated protein kinase PKR enhances transcriptional activation by tumor suppressor p53. Mol. Cell Biol. 19, 2475-2484

$33 \mathrm{Qu}$, L. et al. (2004) Endoplasmic reticulum stress induces p53 cytoplasmic localization and prevents p53-dependent apoptosis by a pathway involving glycogen synthase kinase-3beta. Genes Dev. 18, 261-277

34 Baltzis, D. et al. (2007) The eIF2alpha kinases PERK and PKR activate glycogen synthase kinase 3 to promote the proteasomal degradation of p53. J. Biol. Chem. 282, 31675-31687

35 Bommer, U.A. et al. (2010) Roles of the translationally controlled tumour protein (TCTP) and the double-stranded RNA-dependent protein kinase, PKR, in cellular stress responses. Oncogene 29, 763773

36 Yoon, C.H. et al. (2009) PKR, a p53 target gene, plays a crucial role in the tumor-suppressor function of p53. Proc. Natl. Acad. Sci. U.S.A. 106, $7852-7857$

37 Thaw, P. et al. (2001) Structure of TCTP reveals unexpected relationship with guanine nucleotide-free chaperones. Nat. Struct. Biol. 8, 701-704

38 Susini, L. et al. (2008) TCTP protects from apoptotic cell death by antagonizing bax function. Cell Death Differ. 15, 1211-1220

$39 \mathrm{Li}, \mathrm{F}$. et al. (2001) Characterization of fortilin, a novel antiapoptotic protein. J. Biol. Chem. 276, 47542-47549
40 Zhang, D. et al. (2002) Physical and functional interaction between myeloid cell leukemia 1 protein (MCL1) and fortilin. The potential role of MCL1 as a fortilin chaperone. J. Biol. Chem. 277, 37430-37438

$41 \mathrm{Liu}, \mathrm{H}$. et al. (2005) Stabilization and enhancement of the antiapoptotic activity of mcl-1 by TCTP. Mol. Cell. Biol. 25, 3117-3126

42 Yang, Y.et al. (2005) An N-terminal region of translationally controlled tumor protein is required for its antiapoptotic activity. Oncogene 24, $4778-4788$

43 Chen, S.H. et al. (2007) A knockout mouse approach reveals that TCTP functions as an essential factor for cell proliferation and survival in a tissue- or cell type-specific manner. Mol. Biol. Cell 18, 2525-2532

44 Loo, Y.M. and Gale, M., Jr (2011) Immune signaling by RIG-I-like receptors. Immunity 34, 680-692

45 Ohtani, M. et al. (2010) Evolutional conservation of molecular structure and antiviral function of a viral RNA receptor, LGP2, in Japanese flounder, Paralichthys olivaceus. J. Immunol. 185, 7507-7517

46 Satoh, T. et al. (2010) LGP2 is a positive regulator of RIG-I- and MDA5mediated antiviral responses. Proc. Natl. Acad. Sci. U.S.A. 107, 15121517

47 Vitour, D. and Meurs, E.F. (2007) Regulation of interferon production by RIG-I and LGP2: a lesson in self-control. Sci. STKE 2007, pe20

48 Laplante, M. and Sabatini, D.M. (2012) mTOR signaling in growth control and disease. Cell 149, 274-293

49 Berkowitz, O. et al. (2008) Characterization of TCTP, the translationally controlled tumor protein, from Arabidopsis thaliana. Plant Cell 20, 3430-3447

50 Brioudes, F. et al. (2010) Translationally controlled tumor protein is a conserved mitotic growth integrator in animals and plants. Proc. Natl. Acad. Sci. U.S.A. 107, 16384-16389

51 Wang, X. et al. (2008) Re-evaluating the roles of proposed modulators of mammalian target of rapamycin complex 1 (mTORC1) signaling. $J$. Biol. Chem. 283, 30482-30492

52 Rehmann, H. et al. (2008) Biochemical characterisation of TCTP questions its function as a guanine nucleotide exchange factor for Rheb. FEBS Lett. 582, 3005-3010

53 Dong, X. et al. (2009) Molecular basis of the acceleration of the GDPGTP exchange of human ras homolog enriched in brain by human translationally controlled tumor protein. J. Biol. Chem. 284, 2375423764

54 Burton, J.L. et al. (1994) Specific interactions of Mss4 with members of the Rab GTPase subfamily. EMBO J. 13, 5547-5558

55 Nuoffer, C. et al. (1997) Mss4 does not function as an exchange factor for Rab in endoplasmic reticulum to Golgi transport. Mol. Biol. Cell 8, 1305-1316

56 Itzen, A. et al. (2006) Nucleotide exchange via local protein unfoldingstructure of Rab8 in complex with MSS4. EMBO J. 25, 1445-1455

57 Palamidessi, A. et al. (2008) Endocytic trafficking of Rac is required for the spatial restriction of signaling in cell migration. Cell 134, 135-147

58 Gurdon, J. (2009) Nuclear reprogramming in eggs. Nat. Med. 15, 11411144

59 Grisendi, S. et al. (2006) Nucleophosmin and cancer. Nat. Rev. Cancer $6,493-505$

60 Johansson, H. et al. (2010) Phosphorylated nucleolin interacts with translationally controlled tumor protein during mitosis and with Oct4 during interphase in ES cells. PLoS ONE 5, e13678

61 Johansson, H. et al. (2010) Translationally controlled tumor protein interacts with nucleophosmin during mitosis in ES cells. Cell Cycle 9, $2160-2169$

62 Linares-Cruz, G. et al. (1998) p21WAF-1 reorganizes the nucleus in tumor suppression. Proc. Natl. Acad. Sci. U.S.A. 95, 1131-1135

63 Roperch, J.P. et al. (1998) Inhibition of presenilin 1 expression is promoted by $\mathrm{p} 53$ and $\mathrm{p} 21 \mathrm{WAF}-1$ and results in apoptosis and tumor suppression. Nat. Med. 4, 835-838

64 Roperch, J.P. et al. (1999) SIAH-1 promotes apoptosis and tumor suppression through a network involving the regulation of protein folding, unfolding, and trafficking: identification of common effectors with p53 and p21(Waf1). Proc. Natl. Acad. Sci. U.S.A. 96, 80708073

65 Cans, C. et al. (2003) Translationally controlled tumor protein acts as a guanine nucleotide dissociation inhibitor on the translation elongation factor eEF1A. Proc. Natl. Acad. Sci. U.S.A. 100, 13892-13897

66 Langdon, J.M. et al. (2004) Identification of the interaction between the human recombinant histamine releasing factor/translationally 
controlled tumor protein and elongation factor-1 delta (also known as eElongation factor-1B beta). Biochim. Biophys. Acta 1688, 232-236

67 Bazile, F. et al. (2009) Complex relationship between TCTP, microtubules and actin microfilaments regulates cell shape in normal and cancer cells. Carcinogenesis 30, 555-565

68 Zhang, J. et al. (2012) Role of the translationally controlled tumor protein in DNA damage sensing and repair. Proc. Natl. Acad. Sci. U.S.A. 109, E926-E933

69 Kim, M.J. et al. (2008) Transgenic overexpression of translationally controlled tumor protein induces systemic hypertension via repression of Na+,K+-ATPase. J. Mol. Cell. Cardiol. 44, 151-159

70 Raouf, A. et al. (2012) The biology of human breast epithelial progenitors. Semin. Cell Dev. Biol. 23, 606-612

71 Solozobova, V. and Blattner, C. (2011) p53 in stem cells. World J. Biol. Chem. 2, 202-214

72 Gatza, C.E. et al. (2008) Altered mammary gland development in the p53+/m mouse, a model of accelerated aging. Dev. Biol. 313, 130-141

73 Tyner, S.D. et al. (2002) p53 mutant mice that display early ageingassociated phenotypes. Nature $415,45-53$

74 Jerry, D.J. et al. (2000) A mammary-specific model demonstrates the role of the p53 tumor suppressor gene in tumor development. Oncogene 19, 1052-1058

75 Cicalese, A. et al. (2009) The tumor suppressor p53 regulates polarity of self-renewing divisions in mammary stem cells. Cell 138, 1083-1095

76 Pece, S. et al. (2011) NUMB-ing down cancer by more than just a NOTCH. Biochim. Biophys. Acta 1815, 26-43
77 Colaluca, I.N. et al. (2008) NUMB controls p53 tumour suppressor activity. Nature 451, 76-80

78 Pece, S. et al. (2010) Biological and molecular heterogeneity of breast cancers correlates with their cancer stem cell content. Cell 140, 62-73

79 Telerman, A. et al. Jones Day. Proteic binding partners of TCTP and methods of modulating tumor reversion or cell apoptosis, US 2006/ 0140970 A1

80 Susini, L. et al. (2001) Siah-1 binds and regulates the function of Numb. Proc. Natl. Acad. Sci. U.S.A. 98, 15067-15072

81 Feng, Z. and Levine, A.J. (2010) The regulation of energy metabolism and the IGF-1/mTOR pathways by the p53 protein. Trends Cell Biol. $20,427-434$

82 Takahashi, K. and Yamanaka, S. (2006) Induction of pluripotent stem cells from mouse embryonic and adult fibroblast cultures by defined factors. Cell 126, 663-676

83 Yamanaka, S. (2009) A fresh look at iPS cells. Cell 137, 13-17

84 Lin, T. et al. (2005) p53 induces differentiation of mouse embryonic stem cells by suppressing Nanog expression. Nat. Cell Biol. 7, 165-171

85 Topczewska, J.M. et al. (2006) Embryonic and tumorigenic pathways converge via Nodal signaling: role in melanoma aggressiveness. Nat. Med. 12, 925-932

86 Hardy, K.M. et al. (2010) Regulation of the embryonic morphogen Nodal by Notch4 facilitates manifestation of the aggressive melanoma phenotype. Cancer Res. 70, 10340-10350

87 Pece, S. et al. (2004) Loss of negative regulation by Numb over Notch is relevant to human breast carcinogenesis. J. Cell Biol. 167, 215-221 